\title{
Erratum to: Geochemical modelling, ionic ratio and GIS based mapping of groundwater salinity and assessment of governing processes in Northern Gujarat, India
}

Kumari Rina - Chander Kumar Singh •

P. S. Datta $\cdot$ Neha Singh $\cdot$ S. Mukherjee

Published online: 1 October 2013

(c) Springer-Verlag Berlin Heidelberg 2013

Erratum to: Environ Earth Sci (2013) 69:2377-2391

DOI 10.1007/s12665-012-2067-3

The original article has been published inadvertently with some errors in Table 4 . The corrected Table 4 is given below.

The online version of the original article can be found under doi:10.1007/s12665-012-2067-3.

K. Rina · C. K. Singh · N. Singh · S. Mukherjee $(\varangle)$

School of Environmental Sciences, Jawaharlal Nehru University,

New Delhi 110067, India

e-mail: saumitramukherjee3@gmail.com

C. K. Singh

Department of Natural Resources, TERI University,

Vasant Kunj Institutional Area, New Delhi 110067, India

P. S. Datta

Indian Agriculture Research Institute, New Delhi 110012, India 
Table 4 Correlation matrix of various parameters analysed in post-monsoon season

\begin{tabular}{|c|c|c|c|c|c|c|c|c|c|c|c|c|c|c|}
\hline & $\mathrm{pH}$ & TDS & $\mathrm{EC}$ & $\mathrm{CO}_{3}$ & $\mathrm{HCO}_{3}$ & $\mathrm{SiO}_{2}$ & $\mathrm{~F}$ & $\mathrm{Cl}$ & $\mathrm{NO}_{3}$ & $\mathrm{SO}_{4}$ & $\mathrm{Na}$ & $\mathrm{K}$ & $\mathrm{Ca}$ & $\mathrm{Mg}$ \\
\hline $\mathrm{pH}$ & 1 & & & & & & & & & & & & & \\
\hline TDS & 0.030 & 1.000 & & & & & & & & & & & & \\
\hline $\mathrm{EC}$ & 0.112 & 0.944 & 1.000 & & & & & & & & & & & \\
\hline $\mathrm{CO}_{3}$ & 0.578 & 0.314 & 0.354 & 1.000 & & & & & & & & & & \\
\hline $\mathrm{HCO}_{3}$ & 0.402 & 0.396 & 0.448 & 0.328 & 1.000 & & & & & & & & & \\
\hline $\mathrm{SiO}_{2}$ & 0.018 & 0.027 & 0.035 & 0.043 & -0.117 & 1.000 & & & & & & & & \\
\hline $\mathrm{F}$ & 0.215 & 0.013 & 0.083 & 0.278 & 0.191 & -0.168 & 1.000 & & & & & & & \\
\hline $\mathrm{Cl}$ & -0.021 & 0.913 & 0.840 & 0.191 & 0.203 & 0.095 & -0.068 & 1.000 & & & & & & \\
\hline $\mathrm{NO}_{3}$ & -0.416 & -0.068 & 0.024 & -0.292 & -0.270 & 0.209 & -0.093 & -0.023 & 1.000 & & & & & \\
\hline $\mathrm{SO}_{4}$ & -0.056 & 0.678 & 0.533 & 0.114 & 0.142 & 0.011 & -0.053 & 0.766 & -0.236 & 1.000 & & & & \\
\hline $\mathrm{Na}$ & 0.214 & 0.901 & 0.855 & 0.461 & 0.529 & 0.052 & 0.165 & 0.832 & -0.130 & 0.620 & 1.000 & & & \\
\hline $\mathrm{K}$ & -0.323 & 0.130 & 0.086 & -0.162 & -0.201 & -0.244 & -0.166 & 0.166 & 0.164 & 0.139 & -0.045 & 1.000 & & \\
\hline $\mathrm{Ca}$ & -0.165 & 0.294 & 0.248 & -0.071 & -0.080 & 0.262 & -0.215 & 0.398 & 0.103 & 0.303 & 0.169 & 0.358 & 1.000 & \\
\hline $\mathrm{Mg}$ & -0.149 & 0.627 & 0.589 & -0.015 & 0.069 & 0.102 & -0.246 & 0.731 & 0.059 & 0.534 & 0.439 & 0.287 & 0.713 & 1.000 \\
\hline
\end{tabular}

Bold shows moderate to strong correlation 